\title{
Fabrication of Pt Nanoparticles-polypyrrole Composite for Electrocatalyst
}

\author{
Shigehito DEKI, ${ }^{*}$ Heisuke NISHIKAWA, and Minoru MizUHATA
}

Department of Chemical Science and Engineering, Faculty of Engineering, Kobe University (1-1 Rokkodai-cho, Nada, Kobe 657-8501, Japan)

Received November 26, 2003 ; Accepted March 8, 2004

\begin{abstract}
Preparation of polypyrrole (PPy) containing Pt nanoparticles was attempted. The oxidation of pyrrole into polypyrrole proceeded by $\mathrm{H}_{2} \mathrm{PtCl}_{4}$, simultaneously reducing $\mathrm{Pt}(\mathrm{IV})$ to $\mathrm{Pt}(0)$. The $\mathrm{Pt}$ particles were obtained with average size of 2 $\mathrm{nm}$ and fairly narrow size distribution. Moreover, the maximum content of Pt reached over 20 wt $\%$ without any evidence of aggregation. The optimum $[\mathrm{Pt}] /[\mathrm{Py}]$, for obtaining successful Pt/PPy composite including highly concentrated $\mathrm{Pt}$ and exhibiting high electrical conductivity, was determined at $R=0.6$.
\end{abstract}

Key Words : Platinum Nanoparticles, Polypyrrole, Electrocatalyst, Conductive Polymer Composite, Gas Diffusion Electrode

\section{Introduction}

Conducting polymers (e.g., polypyrrole, polyaniline, and polythiophene) containing dispersed metal (e.g., Pt, $\mathrm{Pd}, \mathrm{Ru}$, and $\mathrm{Sn}$ ) particles showed significant enhancement in catalytic activities toward the oxidation of small organic molecules (e.g., $\mathrm{CH}_{3} \mathrm{OH}$ and $\mathrm{HCOOH}$ ) in comparison with bulk platinum electrodes. ' "' In the major ity of the previously reported research, the catalyst was usually dispersed in electric conducting polymer film by means of electrochemical method.

It is well-known that polypyrrole colloids are produced from aqueous and nonaqueous media. For example, the oxidation of the pyrrole monomer can be carried out rapidly using ferric chloride which has been conventionally used as an agent for preparing "pyrrole blacks" (suspensions of pyrrole polymer particles) in solution. ${ }^{9)}$ Recently, Selvan et al. have reported the novel synthesis method for obtaining Au nanoparticles/conducting polymers composites. ${ }^{5-8}$ This method can produce the composite simply through chemically oxidative polymerization of pyrrole using tetrachloroauric acid $\left(\mathrm{HAuCl}_{4}\right)$. As the polymerization proceeds, the reduction of $\mathrm{Au}$ (III) to $\mathrm{Au}(0)$ occurs simultaneously, which lead to the generation of nano-sized Au particles. This method has a simple one step process and can be easily scaled up to manufacture levels. In recent years, Arms et al ${ }^{10)}$ have described the optimum conditions for the polymerization of pyrrole monomer(Py) in aqueous solutions using $\mathrm{FeCl}_{3}$ as oxidant as follows ;

$$
3 \mathrm{Py}+7 \mathrm{Fe}(\mathrm{III}) \mathrm{Cl}_{3} \rightarrow\left[\begin{array}{ccc}
2 \\
\end{array}\right.
$$

Here, there is one $\mathrm{Cl}^{-}$ion associated with every three pyrrole moieties in the polymer chain.

In this study, preparation of polypyrrole containing Pt nanoparticles was attempted. The oxidation of pyrrole into polypyrrole and the reduction of $\mathrm{Pt}(\mathrm{IV})$ to $\mathrm{Pt}(0)$ proceeded using $\mathrm{H}_{2} \mathrm{PtCl}_{4}$ by the following reaction.

$$
3 \mathrm{Py}+7 / 4 \mathrm{H}_{2} \mathrm{Pt}(\mathrm{IV}) \mathrm{Cl}_{6} \rightarrow\left[\begin{array}{ccc}
2 \\
\end{array}\right.
$$

The structural change of $\mathrm{Pt} /$ polypyrrole composites with varying initial ratio of $\mathrm{Pt}$ ion to pyrrole monomer was characterized. Also, the conductivity was measured by the four-point probe method.

\section{Experimentals}

As the monomer material, high purity pyrrole $(98 \%$, Py) purchased from Aldrich was used. Hexachloroplatinate(IV) acid hexahydrate $\left(\mathrm{H}_{2} \mathrm{PtCl}_{6} \cdot 6 \mathrm{H}_{2} \mathrm{O}\right)$ and iron (III) chloride hexahydrate $\left(\mathrm{FeCl}_{3} \cdot 6 \mathrm{H}_{2} \mathrm{O}\right)$ were obtained from Nacalai Tesque at the highest purity available. All reagents were used without further purification. The synthesis was based on that described in Ref. 7 with minor modification described here. Molar ratio of $[\mathrm{Pt}] /[\mathrm{Py}](=$ $R$ ) ranged from 0.1-1.5 with the constant of $\mathrm{Pt}(\mathrm{IV})$ concentration which was adjusted to achieve the desired platinum to Py ratio. Typically, $\mathrm{H}_{2} \mathrm{PtCl}_{6} 6 \mathrm{H}_{2} \mathrm{O}(217.0 \mathrm{mg}$, $0.42 \mathrm{mmol}$ ) were dissolved into $25 \mathrm{ml}$ of water at ambient temperature, which colored to bright yellow. Fifty micro-liter $(0.72 \mathrm{mmol})$ of Py monomer was added into the $\mathrm{H}_{2} \mathrm{PtCl}_{4}$ solution, and the mixture was stirred at room temperature for $24 \mathrm{~h}$, yielding an opaque black dispersion. The dispersion formed was centrifuged at 12000 rpm for about 10 minutes, and hence the black sediment was obtained. The purification was performed for five times repeatedly until the components soluble in distilled water were completely extracted, until the supernatant became transparent. Finally, the sediment was dried over night at room temperature under vacuum and fine pow- 
der was obtained as sample. Contents of platinum in composites were measured by ICP measurement using SPS 1500 VR (Seiko Instruments Inc.). The samples (1 $\mathrm{mg}$ ) were dissolved in dilute nitrohydrochloric acid solution for ICP measurement. TEM images were recorded with a JEOL JEM-2010 microscope operating at $200 \mathrm{kV}$. The samples for SEM were prepared by placing a drop of the powder dispersion ultrasonically redispersed in water onto a carbon-coated copper grid. In order to confirm the formation of chemical bonding of PPy, IR spectra of the samples were obtained using FT-IR (FT/IR 615 $\mathrm{R}$, Japan Spectroscopic Co.) in diffuse reflectance mode. IR spectra were recorded at $4 \mathrm{~cm}^{-1}$ resolution. Electrical DC conductivity of the compressed pellets of composites was measured by the conventional four-point probe technique (Loresta-GP, Mitsubishi Chemical Co.) at room temperature, where the four points in the sample surface were in line at an equal spacing of $1.5 \mathrm{~mm}$.

\section{Results and Discussion}

As described above, the optimum ratio of platinum ion to Py monomer calculated from the reaction scheme(2) is $\mathrm{Pt}: \mathrm{Py}=7 / 4: 3$; i. e., $R=0.6$, for the polymerization of Py monomer using $\mathrm{H}_{2} \mathrm{PtCl}_{6}$ as the oxidant in aqueous solutions. Accordingly, the $\mathrm{Pt} / \mathrm{PPy}$ composite was prepared on the ratio; $R=0.6$. Within a few seconds of adding the pyrrole to the aqueous solution of $\mathrm{H}_{2} \mathrm{PtCl}_{6}$, the color began to change from yellow to black, which indicate the onset of polymerization and gradually black fine sediment was formed in reaction solution. The formed PPy exhibited the characteristic color called "pyrrole black", which was similar as that of PPy sample prepared by the conventional method using $\mathrm{FeCl}_{3}$.

TEM images of the sediment are shown in Fig. 1. Primary particles of PPy with broad size distribution over the range from $50 \mathrm{~nm}$ to a few hundreds were coagu-

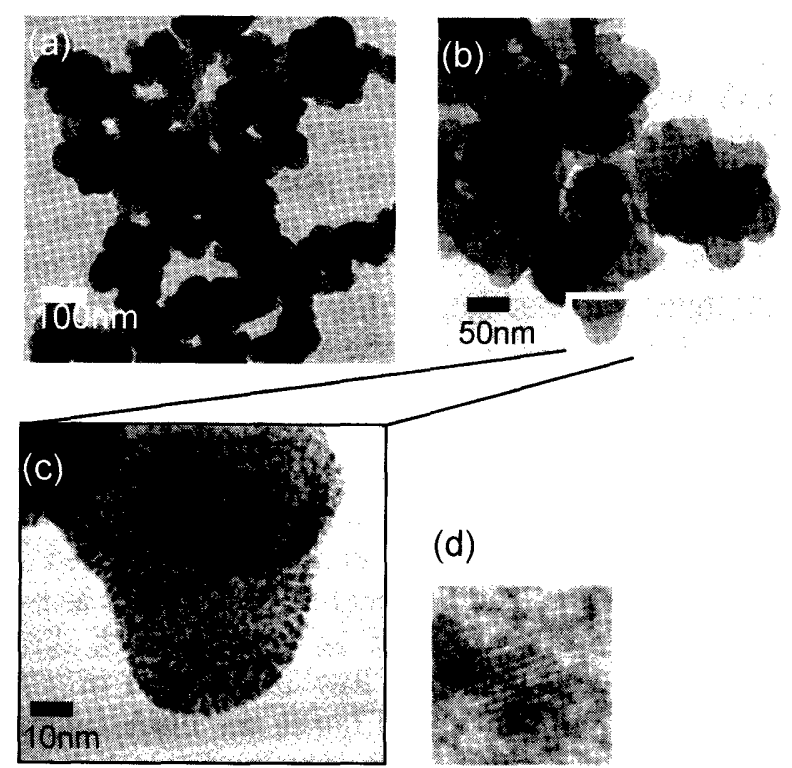

Fig. 1 HR-TEM images of $\mathrm{Pt} / \mathrm{PPy}$ composites for $R=0.6$. (a) and (b) show the morphology of the PPy phase ; (c) Pt supported by PPy, and (d) lattice fringe of supported Pt particle. lated, as shown in Figs. 1a and 1b. Uniformly dispersed Pt nanoparticles with $c a$. $2 \mathrm{~nm}$ in diameter were observed in PPy, as shown in Fig. 1c. Also, visible lattice fringes corresponded to the (111) lattice plane of platinum as shown in Fig. 1d. It is obviously indicated that the sediment is composed of PPy including welldispersed $\mathrm{Pt}$ nanoparticles ( $\mathrm{Pt} / \mathrm{PPy}$ composite).

In the present system, the formation of PPy was also confirmed by the FT-IR measurement. FT-IR spectra of the samples prepared various ratios of $\mathrm{Pt}$ ion to monomer $(R=[\mathrm{Pt}] /[\mathrm{Py}]=0.1,0.3,0.6$, and 1.5) are shown in Fig. 2. In all spectra, the characteristic bipolaron bands attributed to the formation of PPy were observed at 1210 and $930 \mathrm{~cm}^{-1} \cdot{ }^{5-8.11 !}$ These results indicate that PPy is prepared successfully, irrespective of $R$ values. Consequently, it is concluded that $\mathrm{H}_{2} \mathrm{PtCl}_{6}$ successfully acts as oxidant for polymerization of Py.

According to the HRTEM images, Pt nanoparticles can be dispersed in PPy for all samples. From those images, the size distributions were taken as shown in Fig. 3. At $R$ $=0.6$, the mean size of $\mathrm{Pt}$ particles is $2.1 \mathrm{~nm}$, as maxi-

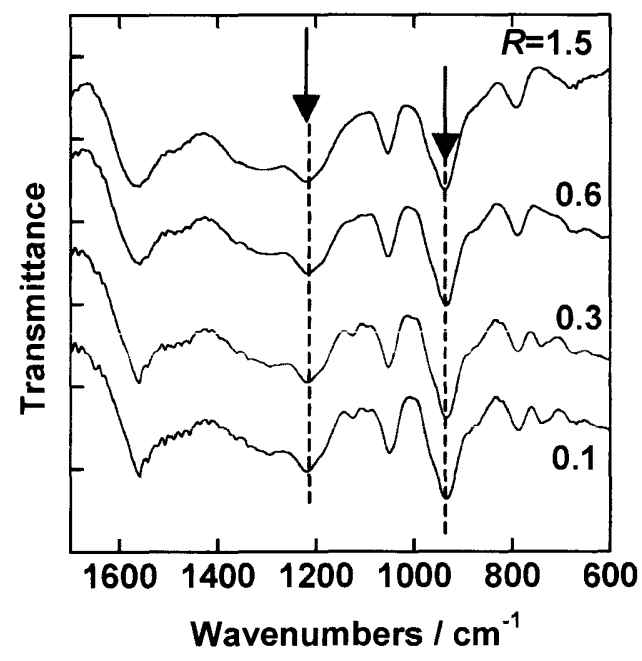

Fig. 2 FT-IR spectra of Pt/PPy composite at the various ratios $; R=0.1-1.5$. Bipolaron bands are given by arrows at $1210 \mathrm{~cm}^{-1}$ and $930 \mathrm{~cm}^{-1}$.

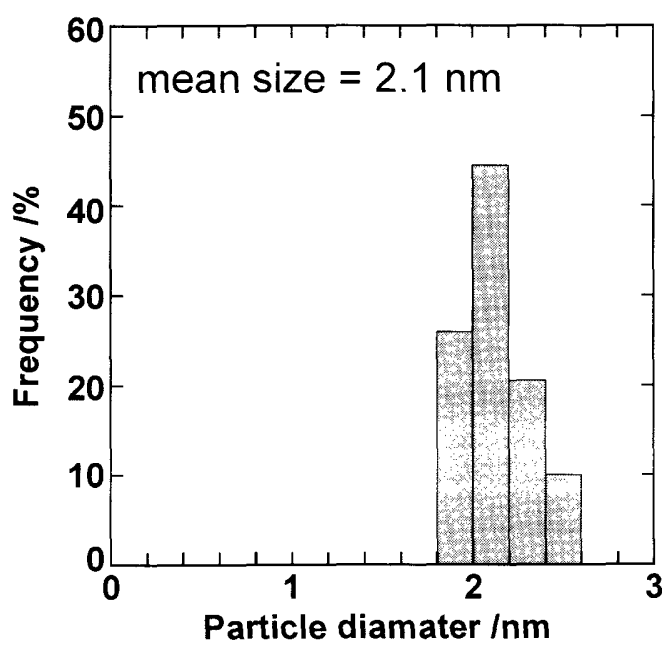

Fig. 3 Size distribution of $\mathrm{Pt}$ particles dispersied in $\mathrm{Pt} / \mathrm{PPy}$ composite at the various ratios $; R=0.6$. 


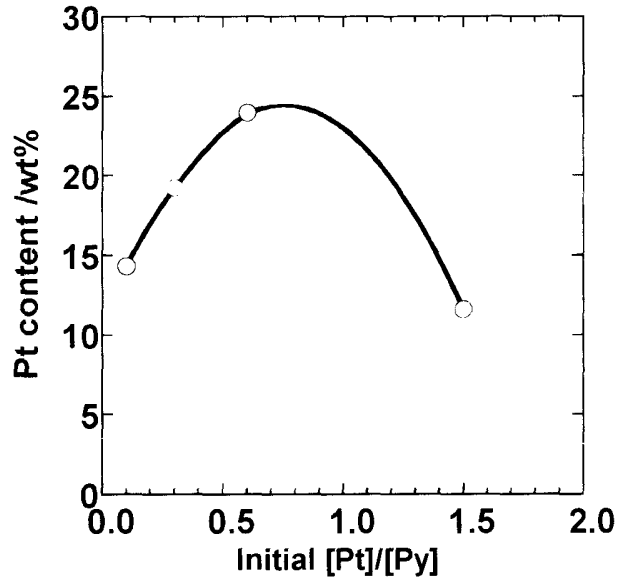

Fig. 4 Variation of Pt content with the initial platinum/pyrrole ratio in the solution.

mum. As Pt concentration decreases relative to $R=0.6$, the mean particle size decreases to $1.7 \mathrm{~nm}(R=0.3), 1.3$ $\mathrm{nm} \quad(R=0.1)$. Meanwhile, when Pt concentration increases, Pt nanoparticles cannot be seen, despite that SEM-EDS Pt element mapping reveals the existence of Pt in the all composite. From EDS spectra, it was found that $\mathrm{Pt}$ content was $c a$. $20 \mathrm{wt} \%$ at $R=0.6$. The inconsistency of these results indicates that Pt particles dispersed in PPy is quite small size such as cluster.

Pt content in the sediment samples was calculated from ICP measurement as shown in Fig. 4. At $R=0.6, \mathrm{Pt}$ content achieved maximum value (24 wt $\%)$, which reveals that highly concentrated Pt nanoparticles dispersed in PPy without aggregation. It is focused that at $R=1.5$. Pt content exhibits minimum value $(12 \mathrm{wt} \%)$, despite the maximum initial concentration of $\mathrm{Pt}$ ion. This tendency correlated with the change of the size of Pt nanoparticles with varying $R$ values. As a result, the formation of $\mathrm{Pt}$ cluster may be due to relatively low concentration of $\mathrm{Pt}$ in the composite. The content and the size of Pt nanoparticles is easily controllable with the initial ratio of $[\mathrm{Pt}] /$ [Py].

The conductivities of the samples were measured using the conventional four-point probe technique. The results were shown in Fig. 5. The highest conductivity of the sample was $R=1.5$. It was $7.7 \times 10^{-4} \mathrm{~S} / \mathrm{cm}$. As confirmed by TEM observation, the size of the corresponding composite $(R=1.5)$ is largest as compared with other samples. According to AC impedance measurement for these sample, each sample showed the resistance without diffusive resistance caused by ionic conduction. Whereas the R-C element caused by the resistance and insulating part was found at the range less than $100 \mathrm{kHz}$ for the samples of $R=0.1$ and 0.3 , the frequency disper-

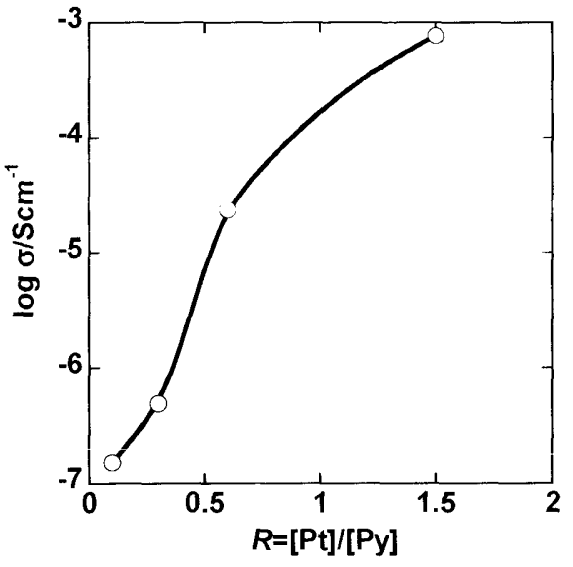

Fig. 5 Variation of electrical conductivity of $\mathrm{Pt} / \mathrm{PPy}$ composite with $R$ at $30^{\circ} \mathrm{C}$.

sion was not observed at $R=0.6$. It is suggested that because the size of coagulation of PPy at $R=0.3,0.1$ is smaller than that of $R=0.6,1.5$, which lead to the fall of conductivity. It is expected that $\mathrm{Pt} / \mathrm{PPy}$ composite obtained by present process shows great promises for future advanced catalytic applications.

\section{Acknowledgments}

This study is supported by Core Research for Evolutional Science and Technology (CREST) of Japan Science and Technology Corporation (JST). The authors would like to thank Professor Masayoshi Okubo and Dr Shuji Fujii of Kobe University for fruitful advice on a preparation of polypyrrole.

\section{References}

1) D. J. Strike, N. F. De Roou, M. Koodelka-Hep, M. Ulmann, and J. Augustynski, J. Appl. Electrochem., 22, 922 (1992).

2) H. Yang, T. Lu, K. Xue, S. Sun, G. Lu, and S. Chen, J. Electrochem. Soc., 144, 2302 (1997).

3) M. Hepel, J. Electrochem. Soc., 145, 124 (1998).

4) K. M. Kost, D. E. Bartak, B. Kazee, and T. Kuwana, Anal. Chem., 60, 2379 (1988).

5) S. T. Selvan, J. P. Spatz, HA. Klok, and M. Moller, Adv. Mater., 10, 132 (1998).

6) S. T. Selvan, Chem. Commun., 1998, 351.

7) S. T. Selvan and M. Nogami, J. Mater. Sci. Lett., 17, 1358 (1998).

8) S. T. Selvan, T. Hayakawa, M. Nogami, and M. Moller, J. Phys. Chem. B, 103, 7441 (1999).

9) M. C. Henry, C. C. Hsueh, B. P. Timko, and M. S. Freund, J. Electrochem. Soc., 148, D155 (2001).

10) S. P. Armes, J. F. Miller, and B. Vincent, J. Coll. Inter. Sci., 118, 410 (1987).

11) B. Tian and G. Zerbi, J. Chem. Phys., 92, 6 (1990). 